\title{
Adopter une perspective cognitive dans \\ l'enseignement de la littérature en FLE avec les apprenants sinophones
}

\author{
Yang $\mathrm{Liu}^{1 *}$ et Wang Zhichao ${ }^{2}$ \\ ${ }^{1}$ Doctorante ED4509, Sorbonne Université, 1 Rue Victor Cousin 75005 Paris, France \\ ${ }^{2}$ Doctorant ED4509, Sorbonne Université, 1 Rue Victor Cousin 75005 Paris, France
}

Résumé. Cet article offre un nouveau point de vue sur l'enseignement de la littérature en didactique du FLE, notamment avec les apprenants sinophones. Il s'agit d'introduire une perspective cognitive dans la lecture littéraire. L'objectif est d'une part, d'établir une interaction et une intercompréhension entre les systèmes langagiers de la langue en cours d'apprentissage et de la langue maîtrisée par les apprenants sinophones, et d'autre part, de construire le sens du texte littéraire, en recourant à la fois aux pensées de l'auteur et au vécu du lecteur. Dans cette optique, les textes littéraires sont considérés comme un support pédagogique où beaucoup de possibilités linguistiques, interculturelles et motivationnelles s'inscrivent. Et pour les apprenants sinophones, ils deviennent aussi un étayage pour surmonter les décalages linguistiques et culturels entre la France et la Chine.

\begin{abstract}
Adopt a cognitive perspective in teaching literature for Chinese-speaking learners of French as a foreign language. This article offers a new point of view on the teaching of literature in the teaching of French as a foreign language, especially for Chinese-speaking learners. It is about introducing a cognitive perspective in literary reading. The objective
\end{abstract}

* Corresponding author : minot8627@gmail.com 
is on the one hand, to establish an interaction and a mutual understanding between the language systems of the target language and the mother tongue of the Chinese-speaking learners, and on the other hand, to build the meaning of the literary text, using the thoughts of the author and the experiences of the reader. In this perspective, literary texts are considered as an educational support where many linguistic, intercultural and motivational possibilities are available. And for Chinese-speaking learners, they also become a support to overcome the linguistic and cultural discrepancies between France and China.

\section{Introduction}

La littérature occupe toujours une position irremplaçable en didactique du français langue maternelle et étrangère et l'enseignement de la littérature a connu de grandes évolutions dans les méthodologies depuis le $\mathrm{XIX}^{\mathrm{e}}$ siècle. Néanmoins, certaines études et mes enquêtes réalisées avec des étudiants sinophones nous montrent que parfois, l'enseignement de la littérature ne reçoit pas des résultats pédagogiques optimaux, à cause de la complexité linguistique, du décalage culturel et de l'impénétrabilité de la métaphore etc. Dans cette optique, les sciences cognitives, travaillant sur «le fonctionnement de l'esprit et du cerveau », nous donnent un nouveau point de vue sur l'enseignement de la littérature dans les cours de FLE. Cet enseignement implique non seulement l'introduction de la linguistique cognitive (Rosch E., 1975 ; Lakoff G. et Johnson M., 1985 ; Langacker R. W., 1987) dans l'analyse des textes littéraires en didactique du FLE, mais aussi une lecture cognitive pour les apprenants sinophones. Cette perspective cognitive permet d'établir une intercompréhension linguistique et culturelle à travers le texte littéraire.

Dans cette étude, je résumerai d'abord brièvement les fonctions de la littérature dans les méthodologies de la didactique de langue maternelle et étrangère en France, ainsi que les situations de l'enseignement de la littérature en didactique du FLE en Chine à travers l'analyse des manuels de français, afin d'identifier les difficultés et les obstacles rencontrés dans l'apprentissage du français avec le texte littéraire. Je mettrai ensuite en lumière la manière de résoudre ces difficultés des apprenants à travers une perspective cognitive, et j'essaierai de les orienter vers une lecture cognitive. Finalement, je proposerai deux approches pédagogiques conçues dans cette étude et présenterai les résultats obtenus dans les cours de FLE.

\section{L'enseignement de la littérature dans les cours de FLE en}




\section{France et en Chine}

\subsection{L'enseignement de la littérature dans les méthodologies de FLE en France}

Le texte littéraire n'est jamais absent dans les méthodologies de langue en France. Les fonctions pédagogiques du texte littéraire sont dégagées au fur et à mesure de l'évolution des méthodologies. Dans la méthodologie dite « grammaire-traduction » utilisée à la fin du XIX siècle, le texte littéraire est considéré «comme matériau qu'il faut analyser pas à pas de manière exhaustive et détaillée pour arriver à la traduction la plus précise et la plus fidèle possible » (Godard A., 2015 : 15). Ainsi, pour réaliser cet objectif, les points grammaticaux et le sens du vocabulaire sont toujours mis en avant dans les cours de langue. Par la suite, dans la méthodologie directe, instaurée de 1880 à 1925 environ, le texte littéraire n'est plus un corpus à traduire, mais «sert de réservoir de formes-le vocabulaire et les constructions grammaticales qu'il contient sont l'objet de la leçon et sont donnés à mémoriser. » (Godard A., 2015 : 18). Il est donc demandé à l'apprenant de comprendre le texte littéraire de manière globale et précise, et de réutiliser les formes qu'il a apprises dans le texte littéraire. Dans les année 30 à 60, la méthodologie active se centre sur le texte littéraire « pour pouvoir aborder les contenus culturels propres à la culture étrangère » (Godard A., 2015 : 19). C'est aussi le premier moment où la compétence relative à la culture est accentuée en didactique du FLE et où la fonction culturelle du texte littéraire est introduite dans l'enseignement. Dans la méthodologie suivante, dite «SGAV », la littérature devient un support visant à développer l'écriture et les connaissances culturelles des apprenants de FLE. De nos jours, les approches communicatives et actionnelles, adoptées la plupart du temps dans les cours de FLE, mettent un accent sur l'authenticité de la littérature.

Le CECRL souligne l'acquisition des compétences relatives à la langue. Ainsi, l'enseignement de la littérature prend en charge de nombreuses compétences langagières de la didactique du FLE. En dehors des compétences en compréhension écrite, productions orale et écrite, il est aussi introduit dans la formation des compétences socioculturelle et sociolinguistique. Par ailleurs, le CECRL met en avant la valeur irremplaçable et la possibilité pédagogique de la littérature dans la didactique du FLE, en évoquant la pluralité de la littérature :

«Les littératures nationale et étrangère apportent une contribution majeure au patrimoine culturel européen que le Conseil de l'Europe voit comme "une ressource commune inappréciable qu'il faut protéger et développer ". Les études littéraires ont de nombreuses finalités éducatives, intellectuelles, morales et affectives, linguistiques et culturelles et pas seulement esthétiques. »(CECRL, 2010 : 47) 
Ces évolutions de la didactique de la littérature en FLE représentent « la richesse polysémique du texte littéraire » (Mimouni L. D., 2010). Les textes littéraires prennent en charge les fonctions pédagogiques suivantes: support des points linguistiques, objet de traduction, corpus culturel, modèle d'écriture, document authentique, expérience esthétique etc. La lecture et l'apprentissage des ouvrages littéraires ont pour but de faire acquérir le sens du texte, qui n'est jamais singulier. L'objectif de l'enseignement de la littérature en France d'aujourd'hui n'est pas de donner un sens prédéfini au texte, mais de découvrir tous les sens possibles qui sont favorables aux compétences relatives à la langue étrangère (Godard A., 2015).

\subsection{Les situations pédagogiques de la littérature dans les cours de FLE en Chine}

La didactique du FLE d'aujourd'hui en Chine est influencée par les méthodologies en vigueur en France, par exemple les approches communicatives et actionnelles sont très à la mode dans beaucoup d'établissements. Néanmoins, en raison des milieux linguistiques différents, les démarches pédagogiques mises en pratique en Chine ne sont pas identiques à celles de la France. Je voudrais donc mettre en lumière les situations de l'enseignement de la littérature dans les cours de FLE à travers l'analyse des manuels de FLE fabriqués et utilisés en Chine, puisque les manuels sont toujours considérés comme un support pédagogique principal dans les cours de FLE en Chine.

La première série de manuels s'intitule Cours de français accéléré (I et II), publié en 1990 par The Commercial Press. Ces manuels sont largement utilisés par les apprenants en FLE qui n'ont pas choisi la langue française comme spécialité à l'université chinoise et dans les institutions de langues. Le premier volume est destiné aux apprenants de niveau débutant, et ne comporte aucun extrait littéraire. Dans le deuxième volume, il existe 12 textes littéraires sur 58 leçons. Cette série de manuels nous montre que d'abord, par rapport à la quantité de texte dans Cours de français accéléré (I et II), les extraits littéraires ne sont pas nombreux. Ensuite, les textes littéraires sont suivis toujours par des explications lexicales et grammaticales en chinois. Finalement, il n'existe pas d'exercices ou bien de questions ouvertes sur l'histoire de ces textes littéraires, il n'y a que des questions relatives au lexique et aux points grammaticaux employés dans le texte.

Mais dans les manuels Le Français, désignant la deuxième série de manuels analysée, la situation des textes littéraires est un peu différente. Le Français inclut quatre manuels, et est édité par Foreign Language Teaching and Research Press en Chine en 1992, l'auteur est Xiaohong MA, un professeur de l'Université de Langues Étrangères de Pékin. Ce manuel est 
rédigé surtout pour les étudiants ayant choisi la spécialité de la langue française dans l'université chinoise. Le programme de chaque leçon ressemble à celui du Cours de français accéléré, à ceci près qu'il ajoute une explication grammaticale ainsi qu'une petite présentation de la civilisation française. Le Français 1 est aussi créé pour les apprenants débutant en FLE, il n'y a pas non plus de textes littéraires dans les leçons, mais à la fin de Le Français 1, l'auteur ajoute une annexe de poèmes (Le Français $1: 374-380$ ), composée de huit poèmes courts. Dans le Français 2, cette annexe de poèmes (Le Français 2 : 427-439) est conservée et augmentée à comporte seize poèmes. En outre, dans les leçons 12 et 13, nous pouvons aussi trouver un extrait de Henri Kubnik, intitulé «Les grands ensembles » (Le Français 2 : 313-315), choisi dans son ouvrage Les Délices des grands ensembles de 1969, et un extrait poétique de Paul Claudel, «Le père " (Le Français 2 : 357), tiré de Feuilles de Saintes, datant de 1925. Les deux derniers manuels, Le Français 3 et 4 sont considérés comme des manuels pour les apprenants de FLE de niveau avancé, l'introduction à la littérature française est très différente de celle dans Le Français 1 et 2. Le Français 3 contient seize leçons parmi lesquelles la moitié utilise au moins un extrait littéraire ; il met aussi huit poèmes dans l'annexe (Le Français 3 : 483-496). Pour Le Français 4, bien qu'il ne comporte plus l'annexe de poèmes à la fin du livre, nous notons la présence de dix leçons parmi lesquelles seize introduisent les extraits littéraires, et ces textes sont beaucoup plus longs que ceux dans les manuels précédents. Au total, dans Le Français 3 et 4, il y a 39 textes littéraires.

Donc, les textes littéraires introduits dans Le Français sont plus divers et plus nombreux que dans le Cours de français accéléré (I et II), mais les manières d'introduire sont similaires, puisque les textes littéraires sont introduits de plus en plus fréquemment au cours de la progression d'apprentissage. Ces deux séries de manuel considèrent donc tacitement que les apprenants de FLE débutants n'ont pas besoin, ou bien ne sont pas capables d'apprendre le texte littéraire. Par ailleurs, les notes des textes littéraires se focalisent toujours sur les explications lexicales en chinois, et les exercices proposés sont généralement en relation avec les points grammaticaux. En fait, sauf les manuels d'origine française, les manuels créés en Chine adoptent majoritairement une méthode de grammaire-traduction. Dans ce sens, les textes littéraires introduits deviennent inévitablement un support des connaissances linguistiques.

\subsection{Les obstacles rencontrés dans l'enseignement de la littérature en didactique du FLE}

Bien que les situations de l'enseignement de la littérature dans la didactique du FLE en 
France et en Chine ne soient pas identiques, il existe certaines questions similaires dans l'enseignement de la littérature en FLE, surtout avec les apprenants sinophones.

Tout d'abord, l'introduction des textes littéraires dans les cours de FLE a pour objectif de donner un contexte authentique pour les unités linguistiques. Néanmoins, dans la réalité, cet objectif pédagogique rencontre beaucoup d'obstacles. Non seulement à cause de la complexité du lexique, de la sémantique et de la syntaxe employée dans le texte littéraire, mais aussi puisque certains phénomènes linguistiques sont très difficiles à illustrer complètement par des extraits littéraires, surtout ceux qui n'existent pas dans le système langagier des apprenants. Par exemple, pour un mot polysémiques, l'un de ses sens, utilisé dans une phrase, ne permet pas aux apprenants de déduire d'autres sens et de les mettre en pratique dans des contextes divers. En particulier, les sens figurés sont plus ambigus pour eux.

Ensuite, le contexte culturel d'un texte littéraire, considéré en quelque sorte comme un atout de ce type de texte, provoque parfois des incompréhensions sur l'intrigue dans l'œuvre littéraire, les sens des locutions et des proverbes, ainsi que les sens du discours sous-jacents. Il n'existe pas une démarche pédagogique qui permet d'établir une interaction et une intercompréhension entre le contexte culturel des apprenants et celui du texte. Ainsi, le décalage-culturel empêche la compréhension de l'œuvre littéraire, et réciproquement, à travers le texte littéraire, il semble très difficile de construire une communication interculturelle.

Finalement, l'analyse esthétique du texte littéraire, introduite parfois dans les cours de FLE, favorise « l'écriture créative » (Baptiste A., 2015) des apprenants de FLE, c'est-à-dire une manière d'écrire où l'« interlangue » de l'apprenant est tout à fait acceptée. Ou bien au contraire, le « savoir-lire » repose beaucoup sur les connaissances obtenues dans la pratique d'écriture (Oriol-Boyer C., 2000). Mais effectivement, la lecture du texte littéraire et l'écriture individuelle sont toujours réduites à une imitation d'écriture sur le sujet ou bien sur la structure de texte. Donc, quelles démarches nous permettent de transférer le savoir-lire du savoir-écrire et réciproquement?

\section{Vers une perspective cognitive dans l'enseignement de la littérature en FLE}

\subsection{La cognition et les sciences cognitives}

Les trois grands obstacles mentionnés dans la partie précédente indiquent que le sens du texte littéraire, les connaissances linguistiques et le système langagier de l'apprenant de FLE ont 
l'air séparés, et que l'apprenant ne peut pas enchaîner l'application et la création dans l'apprentissage du texte littéraire. Dans ce sens, il semble nécessaire d'introduire certaines théories de la cognition applicables à l'enseignement de la littérature en FLE, puisque « la cognition, l'acte de connaître se définit par l'ensemble des processus cognitifs, naturels et artificiels, observables. Elle ne se réduit pas à la connaissance (laquelle se définit comme le résultat produit par un processus cognitif). » (Le Moigne J.-C., 1986 :239) Autrement dit, l'acquisition de la connaissance ne désigne pas la compréhension du système mécanique cognitif. En revanche, si l'apprenant apprend en adoptant «le fonctionnement des connaissances » (Bandura G., $2017:$ 1), il aura plus de capacités pour résoudre les difficultés de compréhension et de pratique dans des situations langagières variables.

Concrètement, que comprennent les sciences cognitives ? Selon Le Moigne (1986 :239), elles couvrent

« champ disciplinaire défini (depuis 1977) de façon autonome par son objet - l'étude des processus cognitifs en général, naturels et artificiels - et par son mode de constitution; l'interaction organisée et organisante d'un certain nombre de disciplines ayant à connaître des processus cognitifs (disciplines relevant tant des "sciences dures" que des "sciences douces") : sciences de la computation et informatique, logique, linguistique, psycholinguistique, psychologie cognitive, neuropsychologie et neurosciences, psychologies sociales [sic] et sciences de la communication, socio-anthropologie, économie, systémique, épistémologie. »

Selon cette définition, les sciences liées majoritairement au « fonctionnement de l'esprit et du cerveau » (Fuchs C., 2004 :1) sont mentionnées, quelles que soient les sciences dures et douces (la linguistique, comprise dans la science sociale et humaine est considérée comme une science douce). Effectivement, les sciences cognitives ne sont jamais isolées, au contraire il existe une relation intrinsèque entre elles. Ce sont les raisons pour lesquelles dans les études de-linguistique cognitive, nous voyons toujours des interactions avec la psychologie, la philosophie, la neurologie etc. Pour mettre en lumière leurs relations, Le Moigne (1986:51) présente le schéma ci-après constitutif des «Sciences de la cognition » (Fig.1.) : 


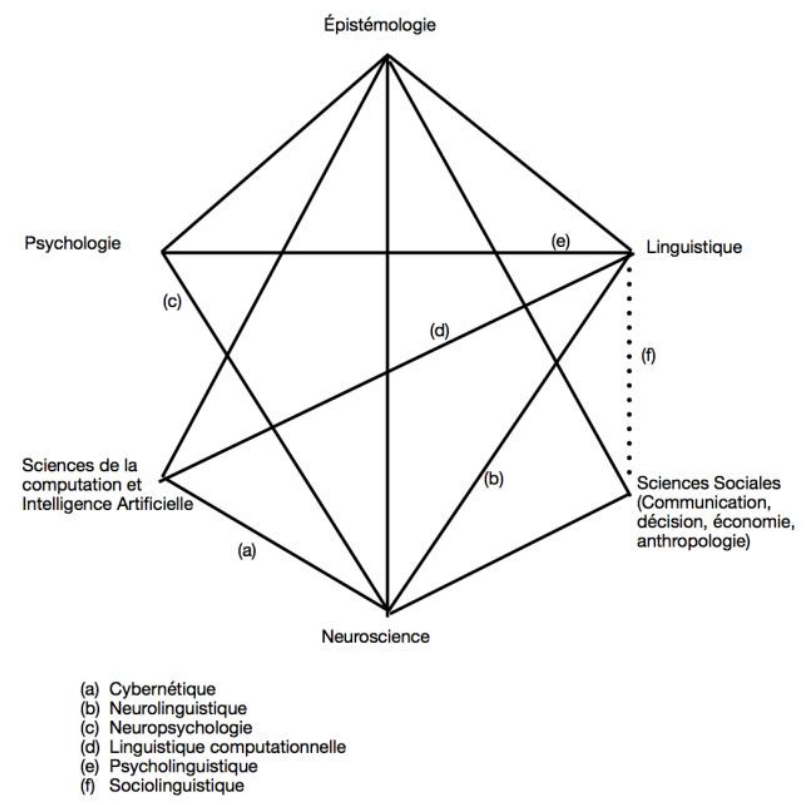

Fig.1. le schéma des « sciences de la cognition »

La linguistique établit un lien avec les six autres disciplines (la sociolinguistique est ici absente, mais la relation entre la linguistique et les sciences sociales est pertinente d'après Rastier F. (1989 : 12)), en formant de nouveaux domaines. Ces sciences de la cognition et leurs relations nous permettent de réfléchir sur la nature de la littérature et sur sa position. La littérature est-elle considérée comme une science cognitive indépendante ? Si oui, quelles sont les relations avec les autres sciences cognitives? Sinon, de quelle manière l'enseignement de la littérature adopte-t-il une perspective cognitive?

\subsection{La littérature et la cognition}

En fait, l'œuvre littéraire, considérée comme « un artefact cognitif » (Bandura G., 2015 : 23) reçoit des critiques lorsqu'elle est associée avec la cognition. Prudhomme (2005) pense que le cognitivisme, introduit dans les études littéraires, aide à appréhender «l'acte d'écriture » et «l'opération de lecture », «[...] chacun de ces processus mobilisant des compétences spécifiques et requérant une chaîne d'opérations mentales que la psychologie cognitive, notamment, devrait pouvoir s'attacher à décrire » (2005 : 93). Mais en réalité, d'une part, à cause de « la spécificité du matériau verbal, son épaisseur signifiante rend en effet caducs les modèles couramment employés pour décrire le processus créateur » (2015:94), et ainsi, l'exploration cognitiviste de l'écrivain et du texte littéraire, est réduite " en général à un 
problème de reconnaissance et de production de forme » (ibid.). D'autre part, les sciences cognitives, désignant un champ interactif de plusieurs disciplines, sont difficiles à explorer dans un texte littéraire : la réalité est que «les tentatives [...] d'exploration 'cognitive' du texte littéraire $[\ldots]$ se passent quasiment de tout recours direct à la psychologie cognitive » (2015 : 95). En outre, selon Bandura (2015), l'association entre la littérature et la cognition se voit reprocher « deux lacunes : premièrement, de ne pas avoir de véritable apport global pour l'étude du texte littéraire et deuxièmement, de recourir à des théories qui restent souvent à l'état d'hypothèse » (2015:35).

En réalité, à cause de la relation compliquée apparaissant dans « la triade auteur-texte-lecteur », ainsi que de «la nature de l'événement littéraire (écriture/lecture) » (Schmitt A., 2012 :145), nous ne devons pas traiter la littérature de manière simple et monosémique.

« Richardson et Steen notent que des représentants majeurs des sciences cognitives ainsi que de domaines connexes ont emprunté allègrement aux études littéraires certaines de leurs notions essentielles: la métaphore pour Lakoff et Johnson, la parabole pour Turner, les scripts et les histoires pour Schank et Abelson, ou encore le stream of consciousness pour Daniell Dennett ; de plus, certains psychologues cognitifs comme Richard Gerrig ont pris pour sujet d'études des textes de fiction. » (Schmitt A., $2012: 146$ )

Ainsi, en didactique du FLE, d'une part, la littérature s'appuie fortement sur la linguistique, puisque l'analyse de textes littéraires doit adopter prioritairement la linguistique cognitive (comme la théorie du prototype de Rosch, la grammaire cognitive de Langacker, les champs métaphoriques de Lakoff et Johnson et la sémantique cognitive de Lakoff etc.) et d'autre part, les spécificités d'œuvres littéraires, décrites par l'esthétique et la littérarité, nous permettent de les analyser par le biais de la poétique cognitive. Autrement dit, cette conception, où la perspective cognitive est introduite dans l'enseignement de la littérature en didactique du FLE, désigne deux types d'études cognitives. L'un est un système linguistique cognitif, désignant une intercompréhension entre le système langagier étranger et celui des apprenants. L'autre est une interaction cognitive entre l'écrivain, le texte et le lecteur, c'est-à-dire, une intercompréhension entre le monde décrit dans l'œuvre, le vécu de lecteur et le monde réel. Dans ce sens, l'apprenant du FLE adopte également un rôle de lecteur.

\subsection{La linguistique cognitive et l'enseignement de la littérature en FLE}

La première partie de cette conception pédagogique concerne un système linguistique cognitif, fondé tout d'abord sur les théories du courant des "grammaires cognitives », 
proposées dans les années 80, et développées par Langacker, Lakoff, Talmy, Fauconnier. Selon eux,

«[...] les deux postulats principaux sont l'existence de mécanismes cognitifs généraux régissant l'activité de langage (au même titre notamment que la perception visuelle ou l'expérience sensori-motrice), et le caractère central de la sémantique (conçue comme une activité de construction de structures symboliques complexes à partir d'unités lexicales et grammaticales). » (Bandura G., 2017 : 11)

C'est-à-dire que d'après les théories des grammaires cognitives, le mécanisme langagier est similaire, quelle que soit la langue. De ce fait, les grammaires cognitives peuvent nous guider dans les cours de FLE pour les apprenants sinophones. Plus précisément, les grammaires cognitives peuvent être résumées par quatre propositions (Desclés J.-P., 1994 : 6-7) :

« Proposition $\mathrm{n}^{\circ}$ 1: Une langue est entièrement descriptible en termes de (i) structures sémantiques; (ii) structures phonologiques; (iii) liens symboliques entre les deux. »

Autrement dit, toutes les langues peuvent être distinguées par ces trois termes. Dans les cours de FLE, nous pouvons établir une comparaison entre le système langagier des apprenants sinophones et celui du français à travers la sémantique dans le texte littéraire.

«Proposition $\mathrm{n}^{\circ} 2$ : La Grammaire Cognitive établit une équivalence entre les structures sémantiques et les conceptualisations (Meaning = Conceptualization). La conceptualisation est considérée comme un processus cognitif, elle doit inclure l'insertion dans un contexte et dans un environnement. La Grammaire Cognitive entre, ici, en conflit avec les représentations de sémantique formelle fondées sur la vériconditionnalité. »

Ainsi, l'apprentissage de la langue ne se détache pas du contexte, puisque le processus cognitif s'inscrit dans la pratique de l'être humain. L'enseignement de la littérature, introduit dans la didactique du FLE, offre ainsi toujours un document authentique, celui du texte littéraire, pouvant donner un contexte réel pour les connaissances linguistiques. Dans cette étude, c'est aussi un support permettant de contextualiser la conceptualisation et les structures sémantiques du français.

«Propositions $n^{\circ} 3: 3.1$. Les structures sémantiques ne sont pas universelles; elles dépendent de chaque langue à des degrés variables; de plus, la structure sémantique est fondée sur une imagerie conventionnelle et relative à diverses structures de connaissances. 3.2. La grammaire ne constitue pas un niveau formel de représentation. La grammaire est une symbolisation conventionnelle des structures sémantiques. 3.3. Il n'y a pas de distinctions significatives entre lexique et grammaire : lexique, morphologie, syntaxe forment un continuum de structures symboliques qui diffèrent 
selon divers paramètres mais peuvent être divisés en composantes séparées relativement arbitraires. »

Depuis la troisième proposition, nous nous apercevons que d'une part, les structures sémantiques de chaque langue se différencient, puisqu'elles sont également conditionnées par les connaissances non linguistiques, variant selon les langues. Ainsi, il faut comprendre les structures sémantiques à l'aide de connaissances culturelles, conventionnelles, artistiques, historiques etc. ; et d'autre part, cette proposition nous explique pourquoi la méthode dite " grammaire-traduction », adoptée par les manuels de français en Chine, présentés dans la partie précédente, ne favorise pas la compréhension du texte littéraire. Comme la grammaire et le lexique désignent des «structures symboliques», résumées selon les innombrables phrases différentes en contexte varié, elles sont donc décontextualisées et abstraites. Ainsi, ce processus de symbolisation peut être considéré comme «un encodage»: quand l'apprenant appréhende ce « code », dans la lecture du texte littéraire, il met en pratique une démarche de « décodage », où beaucoup de facteurs linguistique, non linguistiques, culturels, etc. influencent sa réalisation. Au contraire, la grammaire cognitive nous propose d'observer d'abord les phrases en contexte, puis de déduire les structures sémantiques, en les s'accompagnant des explications grammaticales de l'enseignant. L'apprenant peut donc stocker ces structures sémantiques dans son système d'expression.

«Proposition $n^{\circ} 4$ : Les représentations figuratives sont généralement ignorées dans la linguistique; un cadre conceptuel adéquat pour la linguistique devrait considérer les représentations figuratives comme non pas un problème mais comme une partie de la solution. »

Pour les apprenants sinophones, l'habitude de compréhension et de mémorisation, influencée par les caractéristiques du mandarin et par leurs expériences d'apprentissage de la langue maternelle, dépend fortement de la vision. Ainsi, les représentations figuratives s'adaptent à leurs habitudes d'apprentissage, de compréhension et de mémorisation.

Par conséquent, en didactique du FLE, l'enseignant doit essayer de construire ou bien de retrouver un mécanisme langagier commun déduit à travers la langue étudiée et la langue des apprenants, pour que les sens de certaines unités lexicales et grammaticales compliquées et implicites puissent émerger naturellement dans la pragmatique de langue.

Entre autres, les mots polysémiques posent toujours des problèmes de compréhension chez les apprenants de langue. Dans cette optique, nous pouvons recourir aux relations de catégorisation de la linguistique cognitive :

Première relation de catégorisation : «A schéma $\mathrm{B}[\ldots]$ dans ce cas, $\mathrm{B}$ est entièrement compatible avec les spécifications de A mais il est précisé avec plus de détails (hiérarchisation taxinomique, hyponymie, granularité). Exemple: meuble schéma 
table/chaise/fauteuil. » (Desclés J.-P., $1994: 8$ )

Deuxième relation de catégorisation : " "A protot B" » est proposé selon la théorie du « prototype » de Rosch (1975), désignant une manière de catégoriser surtout lexicale,

« dans ce cas, B entre en conflit, sur quelque(s) unes des spécifications de A, mais il fait partie de la même catégorie en vertu des similitudes perçues ou des relations communes entre A et B. Exemple : voiture protot voiture-à-deux-portes. D'une façon générale, un élément linguistique est polysémique; sa signification est multiple et chaque signification particulière apparaît par des variations contextuelles. » (Desclés J.-P., $1994: 8$ )

La première relation de catégorisation implique une relation hiérarchique du lexique, par exemple le nom collectif comprend un ensemble de personnes ou bien de choses. Il n'établit donc que deux niveaux de hiérarchie. Pour les mots polysémiques, la deuxième relation de catégorisation est plus adaptable, puisque « La principale caractéristique d'un prototype est de partager de nombreuses propriétés avec certains objets du monde (qui forment une catégorie) et peu avec les autres objets (qui de fait appartiennent à d'autres catégories). » (Riccio P.-M., Metge M. et Agostinelli S., 2015 : 6). Ainsi, cette théorie est considérée comme «l'une des grandes sources d'inspiration, qui alimente notamment les nombreux travaux sur la polysémie d'unités lexicales donnant lieu à des représentations géométriques du sens » (ibid.).

Une autre théorie importante en linguistique cognitive pour cette étude concerne la métaphore, relevant de la culture et de la sémantique et difficile à comprendre pour l'apprenant de langue. Elle est définie par Lakoff «comme un mécanisme général de la pensée, massivement présent dans les langues, permettant d'appréhender des concepts abstraits à travers l'expérience sensori-motrice » (ibid.). Bien que cette notion ne soit pas traitée en tant qu'objectif pédagogique principal en didactique du FLE, la métaphore est un concept dont nous dépendons dans la vie quotidienne et les actions et les paroles de l'être humain sont pleines d'emplois métaphoriques (Lakoff G. Johnson M., 1985). Donc, quel que soit le mot polysémique ou la métaphore, ils sont indispensables pour la compétence de communication des apprenants.

\subsection{La poétique cognitive}

À part la linguistique cognitive, l'introduction de la perspective cognitive dans l'enseignement de la littérature en didactique du FLE signifie également une interaction cognitive établie entre les pensées de l'écrivain, les informations du texte littéraire et la compréhension du lecteur. Nous recourons ainsi à la poétique cognitive, désignant une 
approche inédite relative à la littérature, proposée par Tsur. Elle essaie de « rétablir des liens entre le réel et la littérature ; non pas par le biais de la mimesis réaliste, mais par celui de l'expérience du lecteur » (Tsur R., 2012: 143). Ainsi, comme l'ouvrage littéraire est un produit artificiel, il signifie donc une œuvre cognitive et sa création dépend fortement du cerveau humain (Bandura G., $2015: 35$ ).

La poétique cognitive souligne la réception du lecteur dans la construction du sens du texte littéraire :

«La théorie de la réception n'est pas une position critique unifiée conceptuellement, mais un terme qui est maintenant associé au travail de critiques qui utilisent les mots lecteur, processus de lecture, et réception pour délimiter leur champ d'études. [...] Les critiques se réclamant de la théorie de la réception soutiendraient qu'un poème ne peut pas être compris en dehors des résultats qu'il produit. Ses "effets", psychologiques et autres, sont essentiels à toute description précise de son sens, puisque ce sens n'a aucune réalité en dehors de son existence dans l'esprit du lecteur. » (Tompkins J., 1980 :9)

Donc, le sens du texte littéraire n'est pas conditionné seulement par l'auteur, ou bien par le texte lui-même, mais aussi par sa réception, autrement dit par la compréhension et l'interprétation du lecteur. Selon Bandura (2015), dans le processus de la lecture, « le cerveau du lecteur reconstruit le sens du texte littéraire, autrement dit, les mécanismes cognitifs qui permettent de comprendre l'objet artistique qu'est le texte. Le sens, dans ce contexte, se construit autour de deux composants : d'un côté, le texte en tant que stimulus et de l'autre, le cerveau du lecteur, en tant que réponse à ce stimulus. » $(2015: 25)$.

Nous pouvons donc déduire que le sens d'un texte est variable et progressif. « Le "sens" d'une œuvre ne peut être étudié qu'en étendant son analyse au contexte de réception et à la prédétermination du lecteur ; avec deux variantes : ce "sens" est la moyenne de la textualité neutre de l'œuvre (non lue) et de sa lecture / la textualité neutre n'existe pas et seule l'œuvre lue est réelle. » (Schmitt A., $2012: 148)$

En outre, pendant le processus de la création littéraire, l'auteur prend aussi conscience du lecteur. Selon Sartre (1948), l'auteur et le lecteur se complètent mutuellement à travers la lecture, puisque « écrire, c'est donc à la fois dévoiler le monde et le proposer comme une tâche à la générosité du lecteur. C'est recourir à la conscience d'autrui pour se faire reconnaître comme essentiel à la totalité de l'être. » (1948:67) et «c'est l'effort conjugué de l'auteur et du lecteur qui fera surgir cet objet concret et imaginaire qu'est l'ouvrage de l'esprit. Il n'y a d'art que pour et par autrui » (1948 : 55). Autrement dit, d'un côté, l'auteur crée une œuvre littéraire à travers l'écriture, de l'autre côté, le lecteur lit cette œuvre à travers la lecture, leurs consciences et leurs interprétations construisent conjointement le sens du texte, 
étant à la fois multicouche (le sens initial du texte conçu par l'auteur et le sens relativement complet construit par le lecteur), polysémique (les interprétations variables dépendent fortement du vécu de lecteur) et multidisciplinaire (les sens ne s'arrêtent pas à la littérature, mais s'étendent à la culture, la linguistique, la psychologie, la cognition etc.). Ainsi, dans les cours de FLE, l'apprenant, en tant que lecteur, représente un texte littéraire en le liant avec son propre contexte éducatif et culturel, donc, comment traiter et analyser l'interprétation du texte littéraire de l'apprenant dans les cours de FLE?

Conformément aux théories de la poétique cognitive, Schmitt (2012) propose trois grandes catégories d'outils d'analyse, pouvant donner des inspirations pour cette conception pédagogique. Tout d'abord, ce sont les stéréotypes et la mimésis, désignant le mode d'appréhension de la réalité, « les données qui nous permettent d'unifier le réel sont dans un premier temps morcelées dans notre cerveau avant d'être rassemblées et unifiées en types cognitifs. » (Schmitt A., 2012 :150). Ainsi, le lecteur peut toujours mettre en pratique potentiellement des stéréotypes déjà construits, et pendant la lecture, les remplacer constamment par de nouveaux stéréotypes.

«La vie culturelle n'échappe donc en rien à la schématisation du réel, et cette constatation a des implications multiples qui soulignent l'intérêt de la poétique cognitive en ce qui concerne l'analyse quelquefois problématique de la mimesis et du réel dans la littérature. La littérature utilise en effet les cartes cognitives à plusieurs niveaux : dans la représentation du réel, dans l'organisation diégétique, dans la relation intersubjective auteur/lecteur. » (Schmitt A., 2012 :151)

Donc, les stéréotypes interprétés par l'apprenant du FLE à travers une œuvre littéraire sont toujours différents, et ils représentent en quelque sorte sa propre compréhension du monde réel. Par exemple, les apprenants sinophones vivant ou étudiant en France possèdent toujours des stéréotypes différents pour la France de ceux qui habitent toujours en Chine.

Ensuite, bien que dans la partie précédente, nous mettions un accent sur l'interprétation du lecteur, il faut aussi prendre en compte la présence du texte littéraire et ses signes, puisque les mondes textuels sont construits d'une part, par les flux d'information, venant du texte littéraire et d'autre part, par notre mécanisme de mémorisation.

« Le texte fournit les signes qui sont identifiés à l'aide d'une mémoire superposant les schémas narratifs, les conventions paratextuelles et autres types de figure de l'auteur, censés renseigner le lecteur sur l'identité générique d'un texte, par exemple. La compréhension d'un texte s'articule ainsi autour de deux notions essentielles: les attentes du lecteur (anticipation du sens) et la gestion mnémonique du flux d'information (la lecture). » (Schmitt A., 2012 :153)

Selon Schmitt, le lecteur trouve constamment de nouvelles informations (désignant les 
connaissances du monde générales et évolutives) dans une œuvre littéraire, et les stocke provisoirement dans sa mémoire à court terme (Holland N., 2009). Après, les éléments principaux de ces nouvelles informations, qui peuvent modifier "le schéma maître » (Schmitt A., 2012 :155), sont introduits dans sa mémoire à long terme (Holland N., 2009), «l'interaction entre schéma maître et flux d'information apparaissant alors comme le cœur de l'activité herméneutique.» (Schmitt A., 2012 :155). Donc, en s'opposant au structuralisme, travaillant sur la construction d'une œuvre constante, la poétique cognitive considère le récit comme « un organisme idéatif en constante mutation » (Schmitt A., 2012 :155). Par conséquent, bien que les sciences cognitives fassent plus attention au mécanisme cognitif de l'être humain et à ses comportements (auteur/lecteur), il ne faut pas négliger les possibilités du texte littéraire proposées. La nature du texte littéraire, les manières de l'introduire, le découpage du texte, l'ordre d'introduction de certains textes différents etc. influencent les flux d'information et, par suite, les interprétations des apprenants. La poétique cognitive offre un nouvel angle de vue sur le choix, l'analyse et l'enseignement du texte littéraires, autrement dit, nous ne focalisons pas sur la structure figée et prédéfinie du texte, à travers les énormes signes et les éléments variables du texte, nous essayons de faire construire des structures possibles par les lecteurs/les apprenants.

Le troisième outil d'analyse proposé par la conception cognitive concerne les espaces et les zones narratifs dans le monde fictif construits par le lecteur après sa lecture. Généralement, nous pensons que les espaces narratifs sont en quelque sorte prédéfinis par l'auteur et par les signes du texte littéraire. Néanmoins, d'après les études de Marie-Laure Ryan (2003), "la représentation de l'espace diégétique est donc un processus personnel, assez hétérogène même au sein d'une communauté interprétative extrêmement homogène. $[\ldots]$ Chaque lecteur a une culture visuelle qui lui est propre et appréhende l'espace, le monde du récit, à sa manière. » (Schmitt, 2012 :156) Donc, la notion d'espace ici ne signifie pas seulement un lieu concret, mais correspond à une notion spatiotemporelle du monde de l'histoire, et à «un espace interprétatif», dans lequel s'inscrit beaucoup l'imagination du lecteur. Ainsi, associées à notre conception pédagogique, certaines règles communes sur la construction de l'espace narratif ne doivent pas être négligées, mais en même temps, l'enseignant devrait également accepter et apprécier la variabilité de l'espace interprétatif des apprenants, en les conviant à une production créative.

Pour conclure, dans les cours de FLE, le texte littéraire prend en charge deux types de mission : d'une part, à travers toutes les connaissances relatives à la linguistique cognitive, il permet d'établir une interaction cognitive entre le système langagier de la langue française et celui de l'apprenant. D'autre part, la lecture désigne une « activité empirique » (Bandura G., 2015 : 27), et il faut donc encourager et solliciter une interprétation cognitive du sens du 
texte établie entre l'intention de l'auteur, les signes du texte littéraire et la cognition des apprenants. Cette démarche favorise non seulement les compétences langagières des apprenants du FLE, mais aussi leur communication interculturelle, leur plaisir esthétique et leur motivation.

\section{Deux approches conçues pour cette conception}

Comme cette perspective cognitive comprend deux types de mission, l'un est d'établir une interaction entre deux systèmes langagiers, l'autre est de découvrir la variabilité du sens du texte littéraire. J'ai donc conçu donc deux approches correspondantes, et elles sont déjà mises en pratique au fur et à mesure dans des cours de FLE pour des apprenants sinophones.

\subsection{Le schéma cognitif}

La première approche vise à établir une interaction cognitive entre les unités et les faits linguistiques compliqués de ces deux langues, comme les mots polysémiques et la métaphore. Avec l'aide des œuvres littéraires, de leurs traductions en chinois traditionnel, et d'un logiciel de corpus linguistique AntConc, nous pouvons établir un «schéma cognitif» entre deux systèmes langagiers différents.

La morphologie et la syntaxe spécifiques du chinois révèlent un grand décalage avec les autres langues indo-européennes. Contrairement aux langues écrites en alphabet latin, chaque caractère chinois contient trois éléments : un événement, une graphie et un signifiant (Ryjik K., 1983), qui s'accordent. Comme le chinois est une langue où la pragmatique est dominante, le caractère chinois manque de modifications morphologiques et de nature syntaxique, donc, l'usage des mots chinois dépend fortement du contexte (Lu J., 2015). Selon Giroux (2016), c'est sur la langue maternelle que l'apprenant «prend généralement appui pour aborder l'apprentissage d'une autre langue » (2016:64). De plus, l'apprentissage d'une langue étrangère est une procédure d'intégration. Par conséquent, comment intégrer le français dans le système langagier des apprenants sinophones? Il nous faut pour cela recourir à des corpus linguistiques bilingues et comparables, établis par des œuvres littéraires, afin de mettre en pratique des activités pédagogiques associées.

Dans cette optique, j'importe l'œuvre littéraire française et sa version chinoise sur un logiciel, AntConc, pouvant fonctionner dans un milieu plurilingue et traiter parfaitement le chinois traditionnel et le français. À travers ces deux corpus en français et en chinois construits, on peut consulter facilement les fréquences, les occurrences et les contextes des mots, des locutions et des unités linguistiques, en comparant leurs sens et leurs fonctions respectives dans ces deux langues. L'apprenant peut donc déduire l'emploi des unités 
linguistiques compliquées, en y établissant un «schéma cognitif» (Fig.2.).

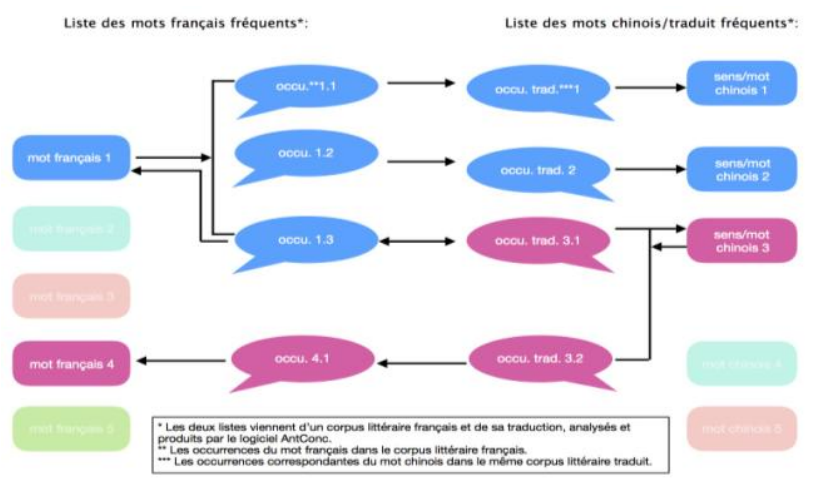

Fig.2. Le schéma cognitif

L'œuvre littéraire mise en pratique dans un cours de FLE est Le Dit de Tianyi de François Cheng (1998), qui raconte une histoire qui se passe en Chine, en français et «à la française », au niveau, non seulement de la langue, mais aussi de la structure et de la représentation symbolique. Le dit de Tianyi peut être considéré comme une biographie : il raconte la vie d'un héros, Tianyi, son enfance et son adolescence en Chine dans les années 30 et 40 (Première Partie : Épopée du départ), les neuf ans vécus en France (Deuxième Partie : Récit d'un détour) et ses expériences après le retour en Chine en 1957 (Troisième Partie : Mythe du retour). En fait, cette histoire ressemble aux expériences de l'écrivain, François Cheng, arrivé en France pour faire ses études quand il était 20 ans. Donc, l'identité de l'écrivain, l'histoire racontée et le mode d'expression sont pleins de caractéristiques interculturelles. Ce sont les raisons pour lesquelles je voudrais proposer une lecture cognitive de cette œuvre. Par ailleurs, les registres de langue de cette œuvre sont variables, et le temps utilisé ne se limite pas au passé simple.

En raison d'une interrogation d'un apprenant sur le sens du mot «pâle », j'ai donc construit un schéma cognitif pour les apprenants sinophones dans un cours de FLE de niveau A2, à travers la deuxième partie de l'œuvre en tant qu'exemple.

Lors de la première étape, nous cherchons le mot «pâle » dans le AntConc, il apparaît cinq fois, et comme les apprenants ont déjà lu et connaissent déjà l'histoire de cette œuvre, nous pouvons facilement trouver les traductions chinoises correspondantes :

Tableau 1. Les occurrences du mot «pâle»

\begin{tabular}{|c|c|c|}
\hline Occurrences en français & $\begin{array}{c}\text { Occurrences en chinois } \\
\text { traditionnel }\end{array}$ & $\begin{array}{c}\text { Résultats de } \\
\text { comparaison }\end{array}$ \\
\hline 1. Dans la lumière gris pâle de & 1. 在巴黎午后的灰光里 & Pâle : dans cette phrase, \\
\hline
\end{tabular}




\begin{tabular}{|c|c|c|}
\hline l'après-midi parisien $[\ldots]$ (p..254) & {$[\ldots](\mathrm{p} .165)$} & $\begin{array}{l}\text { pas de traduction en } \\
\text { chinois. }\end{array}$ \\
\hline $\begin{array}{l}\text { 2. L'un des deux Français qui faisaient } \\
\text { partie du groupe, au visage pâle et à } \\
\text { lunettes, }[\ldots] \text { (p.248) }\end{array}$ & $\begin{array}{l}\text { 2. 和我在同一组的两名法 } \\
\text { 国人中有一个对外特别友 } \\
\text { 善，他面色苍白，戴副眼 } \\
\text { 镜，[...] (p.160) }\end{array}$ & $\begin{array}{l}\text { Pâle: 苍白 (苍 cāng, la } \\
\text { couleur livide et cendrée } \\
\text { de plantes; 白 baí, la } \\
\text { couleur blanche) }\end{array}$ \\
\hline $\begin{array}{l}\text { 3. Allant du rose pâle jusqu'au gris } \\
\text { soyeux durant la journée, le temps se } \\
\text { vêt de pourpre a l'heure du couchant. } \\
{[\ldots](\text { p.203) }}\end{array}$ & $\begin{array}{l}\text { 3. 石块白天里从淡粉直到 } \\
\text { 丝绸似的浅灰, 黄昏时就 } \\
\text { 蒙上了一层紫。(p.129) }\end{array}$ & $\begin{array}{l}\text { Pâle: 淡 (dàn, des } \\
\text { couleurs légères et claires) }\end{array}$ \\
\hline $\begin{array}{l}\text { 4. En parlant, son visage pâle } \\
\text { s'émaciait davantage, cependant que } \\
\text { ses pommettes rosissaient et que ses } \\
\text { yeux se mettaient à briller derrière ses } \\
\text { lunettes, pareils à ceux d'un amoureux } \\
\text { transi. (p.248) }\end{array}$ & $\begin{array}{l}\text { 4. 他说话的时候, 苍白的 } \\
\text { 面孔更细瘦了, 隆起的双 } \\
\text { 㚘却泛起红潮, 眼睛在镜 } \\
\text { 片后闪闪发光, 像热恋中 } \\
\text { 的人在发痴。(p.161) }\end{array}$ & $\begin{array}{l}\text { Pâle: 苍白的 ( identique } \\
\text { avec la deuxième } \\
\text { occurrence; 的 de, } \\
\text { particule grammaticale de } \\
\text { structure) }\end{array}$ \\
\hline $\begin{array}{l}\text { 5. Son visage, d'ordinaire pâle à Paris, } \\
\text { prenait ici la coloration du lieu, une } \\
\text { discrète luminosité légèrement teintée } \\
\text { de rose, avec un reflet qui bleuissait de } \\
\text { temps à autre. (p.267) }\end{array}$ & $\begin{array}{l}\text { 5. 她的脸庞, 在巴黎时总 } \\
\text { 显得苍白, 在这里则染上 } \\
\text { 了当地的颜色, 稍见粉红 } \\
\text { 地暖暖含光, 吹弹得破的 } \\
\text { 皮肤不时辉映着一点血管 } \\
\text { 透出的青蓝。 (p.174) }\end{array}$ & $\begin{array}{l}\text { Pâle: 苍 白 ( identique } \\
\text { avec la deuxième } \\
\text { occurrence ) }\end{array}$ \\
\hline
\end{tabular}

Après la première étape, l'apprenant peut savoir que le mot « pâle » peut être traduit en chinois comme 《苍白》 《淡》, et qu'il qualifie toujours le visage, le teint et la couleur. À la deuxième étape, nous voulons savoir s'il y a d'autres manières pour exprimer les sens comme 《苍白》 en français. Les occurrences de 《苍白》sont au nombre de six, parmi lesquelles trois sont déjà mentionnées dans le tableau $1(2,4,5)$ :

Tableau 2. Les occurrences du mot 《苍白》

\begin{tabular}{|l|l|l|l|}
\hline Occurrences en chinois traditionnel & Occurrences en français & $\begin{array}{c}\text { Résultats de } \\
\text { comparaison }\end{array}$ \\
\hline $\begin{array}{l}\text { 6. 他在中国生活得久了，流泪时的 } \\
\text { 眼睛也依稀带着风眼的形状泪水流 }\end{array}$ & $\begin{array}{l}\text { 6. deux filets de larmes qui } \\
\text { sortaient }\end{array}$ de $\quad$ ses yeux & $\begin{array}{l}\text { 苍 白 的 : d'une pâleur } \\
\text { (n.f.) }\end{array}$ \\
\hline
\end{tabular}




\begin{tabular}{|c|c|c|}
\hline 过他苍白的亚洲型双颊。(p.146) & $\begin{array}{l}\text { devenus en amandes à force } \\
\text { d'avoir vécu en Chine, qui } \\
\text { parcouraient ses joues d'une } \\
\text { pâleur mate, asiatique. } \\
\text { (p.227) }\end{array}$ & \\
\hline $\begin{array}{l}\text { 7. 很细致脸颊有点苍白，但是稍微 } \\
\text { 动就泛起红晕 嘴唇薄而敏锐。 } \\
\text { (p.170) }\end{array}$ & $\begin{array}{l}\text { 7. ces joues plutôt pâles } \\
\text { mais qui deviennent roses à } \\
\text { la moindre émotion ; ces } \\
\text { lèvres minces et sensibles } \\
\text { faites pour moduler les sons. } \\
\text { (p.259) }\end{array}$ & $\begin{array}{l}\text { 苍 白： pâles (adj. pl., } \\
\text { puisque « ces joues » sont } \\
\text { pluriels) }\end{array}$ \\
\hline $\begin{array}{l}\text { 8. 这位共产党员的苍白后来也蔓延 } \\
\text { 到我身上了。(p.161) }\end{array}$ & $\begin{array}{l}\text { 8. La pâleur passionnée du } \\
\text { communiste finit par } \\
\text { déteindre sur moi. (p.249) }\end{array}$ & 苍白： la pâleur (n.f.) \\
\hline
\end{tabular}

La nature des mots chinois n'est pas évidente, elle dépend fortement du contexte. Comme le mot chinois «苍白» peut être utilisé en tant que nom (occurrence 8) et adjectif (occurrence 1-7), cette démarche favorise une distinction des fonctions de nom et d'adjectif chinois, en s'appuyant sur les mots français correspondants, puisque les emplois de nom et d'adjectifs chinois et français sont parfois similaires, comme dans l'occurrence 8 ; de plus, la fonction complément de nom «de + nom » correspond quelquefois à un adjectif (l'occurrence 6 : « ses joues d'une pâleur mate, asiatique » puisqu'elle est traduite en chinois par 《他苍白的亚洲型双顿 »/ 《ses joues pâles et asiatiques »). Par ailleurs, les apprenants sinophones connaissent également l'accord de l'adjectif et du nom (le nom « le visage » s'accorde avec l'adjectif singulier « pâle », mais le nom « ses joues », étant pluriel, s'accorde avec l'adjectif pluriel «pâles »).

Comme l'adjectif "pâle» peut aussi être traduit en chinois par «淡 », («légères et claires »), dans la troisième étape, nous cherchons donc le mot chinois «淡 » dans AntConc, et trouvons six phrases comprenant le mot «淡», mises dans la tableau 3, sauf la dernière phrase qui est déjà citée dans la tableau 1 , occurrence 3 :

Tableau 3. Les occurrences du mot «淡» 


\begin{tabular}{|c|c|c|}
\hline $\begin{array}{l}\text { Occurrences en chinois } \\
\text { traditionnel }\end{array}$ & Occurrences en français & $\begin{array}{l}\text { Résultats de } \\
\text { comparaison }\end{array}$ \\
\hline $\begin{array}{l}\text { 9. 我渴望它, 像渴望一 } \\
\text { 个生命实质, 它可以填满 } \\
\text { 我胃里这个无时或已的 } \\
\text { 饥饿感一一所有日复一 } \\
\text { 日尽义务地咽下的淡无 } \\
\text { 味的食物都已宣告没有 } \\
\text { 满足的能力。(p.168) }\end{array}$ & $\begin{array}{l}\text { J'en avais faim comme d'une substance } \\
\text { vitale qui remplirait ce creux constant que } \\
\text { je ressentais au milieu de mon estomac, } \\
\text { qu'aucun de ces aliments insipides } \\
\text { ingurgités jour après jour par devoir ne } \\
\text { parvenant à combler. (p.257) }\end{array}$ & $\begin{array}{l}\text { 淡无味 ( 淡 dàn léger; } \\
\text { 无 wú sans; 味 weì } \\
\text { goût) : insipides (adj. pl.) }\end{array}$ \\
\hline $\begin{array}{l}\text { 10. 在他的第二任妻子 } \\
\text { 海德莉克的眼神里, 我看 } \\
\text { 到母亲压抑着不安的慈 } \\
\text { 爱和忧虑, 现在母亲的形 } \\
\text { 象在我记忆中逐渐焱化, } \\
\text { 我想念她时, 不免透过这 } \\
\text { 张画像去追忆。(p.143) }\end{array}$ & $\begin{array}{l}\text { Dans les yeux d'Hendrickje, sa seconde } \\
\text { épouse, je reconnaissais la douleur } \\
\text { inquiète et la transparence teintée de } \\
\text { mélancolie de ma mère, au point que je } \\
\text { ne pouvais plus penser à elle, à présent } \\
\text { que son image s'estompait de plus en } \\
\text { plus dans ma mémoire, qu'à travers la } \\
\text { figure de celle-ci. (p.223) }\end{array}$ & $\begin{array}{l}\text { 淡化 (淡 dàn léger; 化 } \\
\text { huà changer/transférer) : } \\
\text { s'estomper (v. } \\
\text { pronominal) }\end{array}$ \\
\hline $\begin{array}{l}\text { 11. 它只好从自身汲取黯 } \\
\text { 淡的光源。(p.165) }\end{array}$ & $\begin{array}{l}\text { Elle était réduite à puiser en elle-même } \\
\text { sa sombre clarté. (p.254) }\end{array}$ & $\begin{array}{l}\text { 黯淡的 (黯 àn obscure; } \\
\text { 淡 dàn léger; 的 de } \\
\text { particule grammaticale de } \\
\text { structure) }\end{array}$ \\
\hline $\begin{array}{l}\text { 12. 各式色香味俱全的 } \\
\text { 小菜, 和船上令人嫌恶的 } \\
\text { 大锅菜大相径庭, 谈话在 } \\
\text { 汤面冒上来的蒸气中热 } \\
\text { 闹地展开, 冲淡了不少墙 } \\
\text { 能里的油垢和霉味, 给人 } \\
\text { 重回故乡的短假象。 } \\
\text { (p.125) }\end{array}$ & $\begin{array}{l}\text { Les conversations animées et les } \\
\text { vapeurs appétissantes qui montaient des } \\
\text { soupes aux nouilles et des plats aussi } \\
\text { colorés qu'épicés, si différents de la } \\
\text { nourriture rébarbative servie sur le } \\
\text { bateau, contribuaient à chasser l'odeur } \\
\text { de graisse moisie tapie dans les fentes } \\
\text { des murs, à répandre une éphémère } \\
\text { illusion du pays natal retrouvé. (p.196) }\end{array}$ & $\begin{array}{l}\text { 冲 淡 ( 冲 chōng laver } \\
\text { avec grande eau/ sortir ou } \\
\text { franchir avec force ; 淡 } \\
\text { dàn léger ; 冲淡 prend les } \\
\text { sens de diluer ou bien } \\
\text { d'affaiblir) : chasser (v.) }\end{array}$ \\
\hline $\begin{array}{l}\text { 13. 未料巴黎的街道如 } \\
\text { 此黯淡, 让我大为惊讶, } \\
{[\ldots](\mathrm{p} .124)}\end{array}$ & $\begin{array}{l}\text { Je fus frapper par la couleur terne des } \\
\text { rues de Paris, }[\ldots](\text { p.195) }\end{array}$ & $\begin{array}{l}\text { 黯淡(黯 àn obscure; 淡 } \\
\text { dàn léger) : terne (adj.) }\end{array}$ \\
\hline
\end{tabular}

Les résultats de la troisième étape nous montrent que le mot chinois «淡 » peut être 
exprimé par les adjectifs français comme « insipide », « sombre », « terne » et «pâle », mais aussi par les verbes français «s'estomper » et « chasser ». Avec le contexte, les apprenants sinophones peuvent établir facilement une intercompréhension entre 《淡» et les mots français correspondants. En fait, les résultats de ces trois tableaux peuvent établir le schéma cognitif suivant (Fig. 3) :

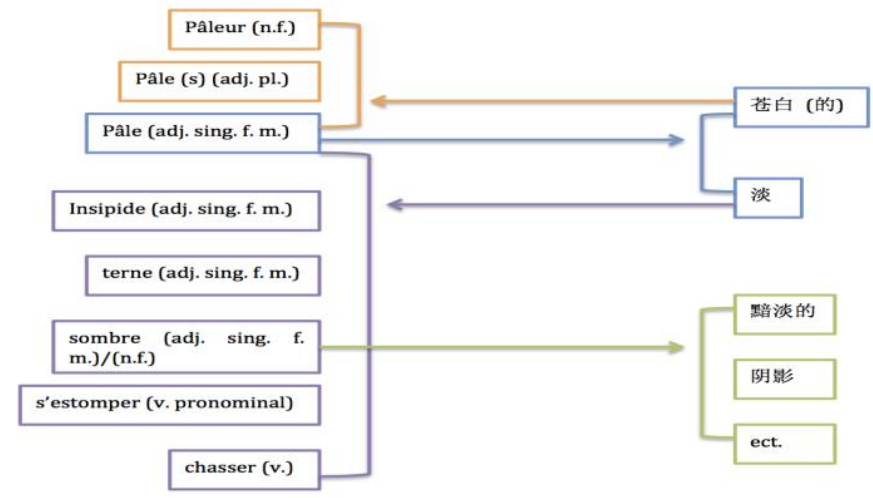

Fig.3. Schéma cognitif construit par le mot " pâle »

Dans la première approche, le schéma cognitif ne se limite pas aux trois étapes, et les apprenants peuvent continuer à le construire selon leurs besoins. Je leur donne le logiciel et les textes de l'œuvre, afin de leur permettre de construire leur propre schéma cognitif à la maison, et dans le cours, je les invite à présenter les schémas en expliquant quels problèmes sont résolus selon ce schéma. Les résultats sont très prometteurs. La plupart des apprenants analyse les différents sens des mots polysémiques, les autres se focalisent sur des points grammaticaux différents entre le français et le chinois, comme l'article français, le qualificateur chinois, la préposition etc. Quelques apprenants sinophones établissent un schéma cognitif entre la préposition «de» et la particule grammaticale de structure en chinois «的» (de, dont la prononciation 的 est identique à celle de la préposition française « de »).

Comme le corpus peut aussi être modifié selon leurs intérêts, cette approche favorise l'autodidactique et la construction de la motivation d'apprentissage des apprenants sinophones, puisqu'ils peuvent établir une interaction entre leur langue maternelle et la langue cible, en introduisant le français dans leur propre système langagier, tout en s'adaptant à leurs besoins. 


\subsection{Les productions écrites et orales des traces de lecture}

La deuxième approche concerne un exercice cognitif écrit et oral, s'appuyant sur les textes littéraires. Comme nous en avons déjà parlé dans la deuxième partie, le sens du texte littéraire résulte de la coopération entre l'écrivain et le lecteur. Le monde créé par l'auteur n'est pas identique à celui du lecteur, puisqu'il se base sur les expériences, les points de vue et les pensées de l'auteur et du lecteur étant parfois partagés et éveillant un écho, mais se différenciant parfois et se complétant. Par ailleurs, selon mes expériences d'enseignement, et mes recherches sur la motivation d'apprentissage des apprenants de FLE, je découvre que les sujets pouvant susciter l'envie de communication chez les apprenants de FLE ont toujours une relation avec leur propre vécu. Par exemple, j'ai proposé deux sujets de communication pour les apprenants de FLE, « Quelles spécialités de France connaissez-vous ? » et « Quelles sont les spécialités de votre pays natal? ", les apprenants montent plus d'intérêt pour le deuxième sujet.

Par conséquent, pour donner une grande liberté d'expression, l'enseignant ne doit pas prédéfinir le sujet de production. Dans cette approche, nous encourageons les apprenants à laisser des traces de lecture, c'est-à-dire que pendant le processus de lecture, ils peuvent enregistrer leurs pensées, sentiments, souvenirs, échos, questions etc. en utilisant des intermédiaires variables, comme le dessin, le brouillon, les notes en français ou bien en chinois. Après, l'enseignant invite les apprenants à parler de ces traces de lecture.

J'ai mis en pratique séparément cette approche pour deux classes de FLE dans une université chinoise et dans une école de langue à Paris. Ces deux classes ont une vingtaine d'apprenants sinophones de niveau A2. Je leur donne les textes français et chinois de la deuxième partie de Le Dit de Tianyi, puisqu'elle raconte les aventures du héros de roman quand il arrive à Paris. C'est une histoire forte où les deux cultures, chinoise et française, se rencontrent, en provoquant des chocs culturels. Pour la première classe de FLE, celle de l'université chinoise, les traces de lecture des étudiants montrent plutôt leurs incompréhensions et leurs interrogations sur la vie de Paris décrite par l'auteur. Je résume les résultats principaux dans le tableau suivant (Tableau 4) :

Tableau 4. Les traces de lecture de la classe 1

\begin{tabular}{|l|l|}
\hline Incompréhensions et & - Qu'est-ce c'est le « Quartier latin »? \\
questions & - Même aujourd'hui, en France, le médecin doit-il venir à la maison pour \\
& soigner le malade? \\
& - Le restaurant pour étudiants de France ressemble-t-il à la cantine de \\
& l'université ?
\end{tabular}




\begin{tabular}{|l|l|}
\hline & - Où sont les quartiers populaires à Paris ? \\
& - Les appartements de France ne sont pas insonorisés. Pourquoi l'auteur \\
& peut-il entendre les bruits de voisins? \\
& - Qui est Rembrandt? \\
& - etc. \\
\hline Rappels et souvenirs & - les expériences d'achat d'un croissant dans une boulangerie chinoise. \\
& - Certains points de vue sur les tableaux de Monet et Van Gogh. \\
& - Le voyage en France, les visites du musée du Louvre, de la Tour Eiffel, \\
& de la Seine etc. \\
\hline
\end{tabular}

Comme les apprenants sinophones de la deuxième classe ont déjà vécu en France, ils comprennent parfaitement la vie de Paris racontée par l'auteur, en permettant de se rappeler leurs propres expériences. Les sujets représentatifs sont résumés dans le tableau suivant (Tableau 5):

Tableau 5. Les traces de lecture de la classe 2

\begin{tabular}{|c|c|}
\hline $\begin{array}{l}\text { Rappels et } \\
\text { échos }\end{array}$ & $\begin{array}{l}\text { - Le décalage entre le Paris réel et l'imagination. } \\
\text { - les stéréotypes sur la Chine et les Chinois chez les Français. } \\
\text { - Les endroits familiers : le quartier latin, Montparnasse, la Seine... } \\
\text { - Les apprenants qui travaillent sur la peinture sont d'accord avec les points de } \\
\text { vue sur les différences entre la peinture chinoise et française. } \\
\text { - Les expériences de travail au cours du séjour en France. } \\
\text { - Les relations avec les voisins. } \\
\text { - Les expériences de voyage en Hollande et en Italie. } \\
\text { - Les expériences de consultation de médecin. } \\
\text { - Les démarches administratives. } \\
\text { - La nostalgie. } \\
\text { - etc. }\end{array}$ \\
\hline
\end{tabular}

Par ailleurs, je les encourage à souligner des phrases qu'ils aiment ou qu'ils vont utiliser dans leurs propres créations. Je cite une partie des phrases soulignées par les apprenants (tableau 6) :

Tableau 6. Les phrases soulignées par les classes 1 et 2

Les phrases et - - «e me sentais pris dans les rets de la ville-labyrinthe. » (p.202)/我犹如陷入 


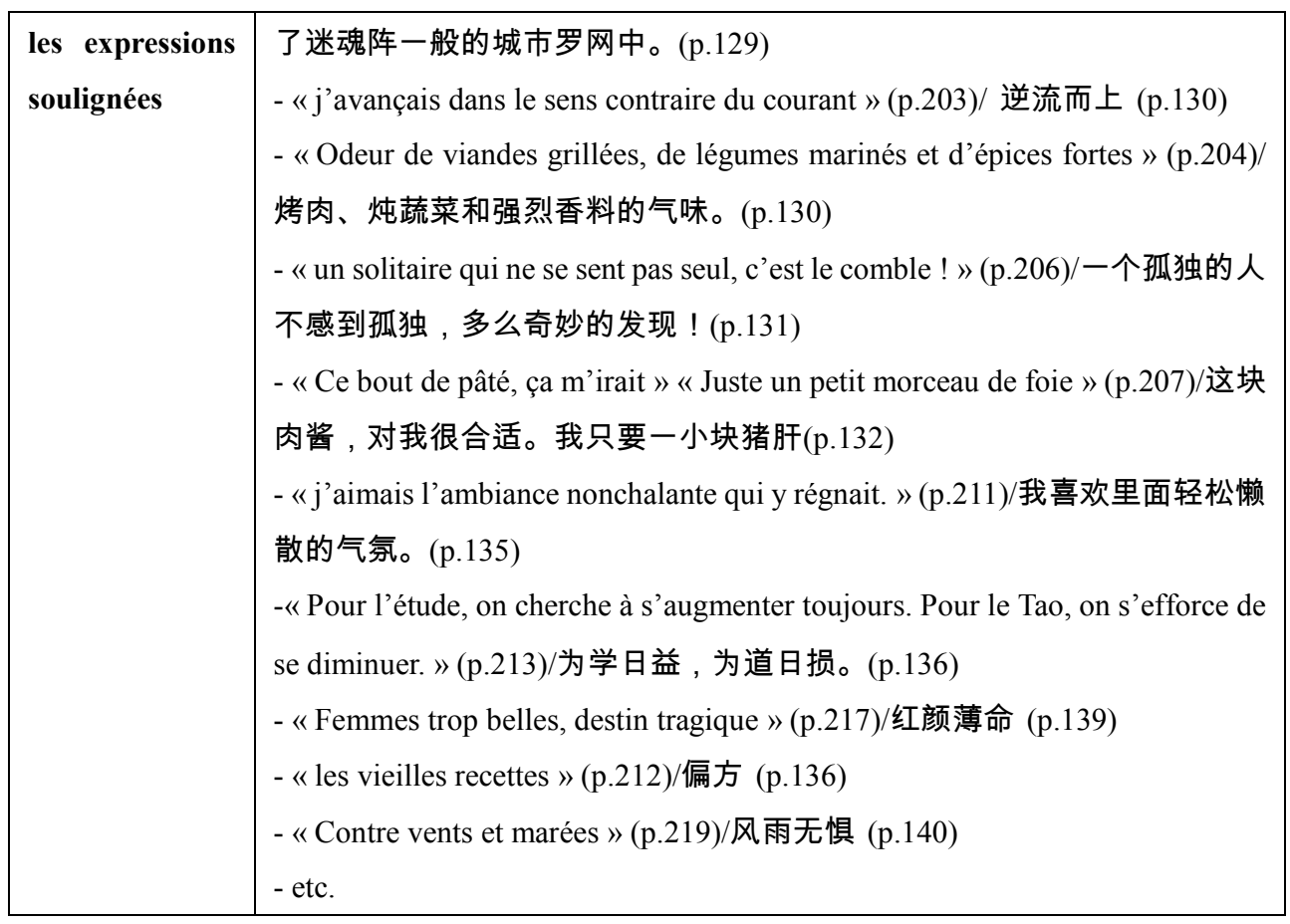

Selon les résultats, nous pouvons nous apercevoir que les productions écrites et orales des apprenants sont liées étroitement avec leur propre vécu et leur contexte culturel, même pour les phrases soulignées, et que les expressions françaises correspondant aux proverbes chinois sont toujours mises en avant. Cette approche introduit les réflexions et la cognition des apprenants dans l'interprétation du texte littéraire, en suscitant leur envie d'échange. Bien que les apprenants sinophones de niveau A2 ne produisent pas beaucoup de phrases longues, correctes et recherchées, l'enjeu est de leur faire raconter leur vie et leurs expériences en français ; les nouveaux mots appris dans le texte littéraire sont alors reproduits facilement par les apprenants.

\section{Conclusion}

Les caractéristiques linguistiques et non linguistiques de l'œuvre littéraire supposent que la perspective cognitive introduite en didactique du FLE désigne deux types d'approche, l'un lié à la linguistique cognitive et l'autre à la poétique cognitive. L'introduction de la cognition dans la linguistique est récente, mais a beaucoup d'adeptes. Néanmoins, la combinaison de la cognition et de la littérature tâtonne, donnant lieu à des redites, des confusions et des contestations, puisque si nous pouvons définir et décrire explicitement ce qu'est la linguistique française ou bien chinoise et ce qu'elle comprend, il n'est pas facile de 
caractériser la littérature française ou chinoise. Ainsi, au lieu de dire que cette étude veut introduire «la littérature cognitive » dans la didactique du FLE, nous préférons adopter l'expression de "perspective cognitive », prenant en compte tous les mécanismes cognitifs de l'auteur, du lecteur, des informations du texte littéraire, ainsi que de la langue utilisée. Les approches pédagogiques que je propose tournent autour de cette conception.

Évidemment, ces deux approches ne sont que des premières tentatives de cette étude. Elles doivent encore être améliorées au fur et à mesure dans la pratique pédagogique. Mais les résultats obtenus nous permettent d'orienter vers des réflexions sur des questions Quels types de textes littéraires sont introduits préférablement ? Comment adapter ces approches aux publics différents (les étudiants à l'université chinoise ou dans une école de langue, les apprenants sinophones habitant en France, etc.) ? De quelles manières ces approches peuvent-elles être mises en pratique dès le début de l'apprentissage du FLE ?

Comme le CECRL essaie de redéfinir la notion de maitrise d'une langue étrangère, en pensant que «l'usager et l'apprenant d'une langue » sont considérés «comme des acteurs sociaux » et que « les ressources cognitives, affectives, volitives et l'ensemble des capacités que possède et met en œuvre l'acteur social » doivent également être prises en compte en didactique du FLE (2001 : 15) cette étude contribue aussi à donner un nouveau rôle aux apprenants sinophones dans les cours de FLE, non seulement un rôle d'apprenant face à un document pédagogique authentique, mais aussi de lecteur natif d'une œuvre littéraire. De ce fait, la perspective cognitive favorise l'adoption du rôle de lecteur pour les apprenants, puisqu'elle ne représente pas seulement les mécanismes cognitifs communs d'un peuple, mais aussi ceux des individus. Cette étude est donc centrée sur la cognition de chaque apprenant sinophone, dans laquelle les enjeux plurilinguistiques, motivationnels et interculturels sont également découverts.

\section{Références bibliographiques}

Bandura G. (2015). La littérature et les sciences cognitives : tableau synthétique d'une approche possible. Acta Romanica, (Tomus XXIX), Szeged : JatePress.

Bandura G. (2017). Littérature et sciences cognitives : apports et légitimité d'une lecture transversale. Carnet, URL : http://journals.openedition.org/carnets/2113.

Baptiste A. (2015). La rôle de la littérature dans les apprentissages langagiers : de l'écriture créative à la conscience de la langue, La littérature dans l'enseignement du FLE (Godard, A. dir.), Paris : Didier, 170-208. 
Cadre Européen Commun de Référence pour les Langues : Apprendre, Enseigner, Evaluer (2001), Strasbourg : Unité des Politiques Linguistiques.

Cheng F. (1998). Le dit de Tianyi. Paris : Albin Michel.

Cheng F. (2009). Tianyi yan. Yang N. (trad.). Pékin : people's Literature Publishing House.

Daunay B. (2007). État des recherches en didactique de la littérature. Revue française de pédagogie, $N^{\circ} 159,139-189$.

Desclés J. P. (1994). Réflexions sur les Grammaires Cognitives. Modèles Linguistique. URL: http://alic.paris-sorbonne.fr/PUBLICATIONS/descles/modeles94.pdf

Fuchs C. (2004). Pour introduire à la linguistique cognitive. La linguistique cognitive, Paris : Ophrys.

Giroux L. (2016). La place et le(s) rôle(s) de la langue maternelle des apprenants en cours de langue étrangère, Synergies France, $n^{\circ} 10,55-68$.

Godard A. (2015). La littérature dans l'enseignement du FLE. Paris : Didier.

Holland N. N. (2009). Literature and the Brain. Gainesville : The PsyArt Foundation.

Lakoff G. et Johnson M. (1985). Les métaphores dans la vie quotidienne. traduction de Defornel M.et Lecercle J.-J., Paris : Minuit.

Langacker R. W. (1987). Foundation of Cognitive Grammar. California : Stanford University Press.

Le Moigne J. L. (1986a). Mécanismes de l'intelligence, intelligence des mécanismes. Paris : Fayard.

Lu J. (2015). Hànyŭ ‘tèdiăn'zhīwǒjiàn (les caractéristiques du chinois à mes yeux). Journal of Chiniese studies. 15-26,

Ma X. (1992-1993). Le Français 1-4, Pékin : Foreign Language Teaching and Research Press.

Mimouni L. D. (2010). La motivation des lycéens algériens en lecture littéraire: l'utilisation de la paralittérature. thèse en Didactique des langues étrangères. Université d'Oran (dir. SARI Fewzia).

Oriol-Boyer C. Conférence sur «Les écritures d'invention » le 13/12/2000 dans un collège de l'académie de Versailles (à Neuilly). https://lettres.ac-versailles.fr/IMG/pdf/Conference_de_Claudette_Oriol.pdf.

Rastier F. (1989). Linguistique et recherche cognitive. Histoire Épistémologie Langage, Science du langage et recherches cognitives, tome 11, fascicule 1, 5-31. 
Riccio P-M., Metge M., Agostinelli S. (2015). La théorie des prototypes comme outil méthodologique pour l'analyse des situations. Les écosystèmes numériques et la démocratisation informationnelle: Intelligence collective, Développement durable, Interculturalité, Transfert de connaissances, Schoelcher.

Prudhomme S. (2005). Littérature et sciences cognitives. Labyrinthe, $n^{\circ} 20$, 93-97. URL: http://journals.openedition.org/labyrinthe/761.

Rosch E. (1975). Cognitive representation of semantic categories. Journal of Experimental Psychology General, vol. 104.

Ryan M. L. (2003). Cognitive maps and the construction of narrative space. Narrative Theory and the Cognitive Science, 214-242.

Ryjik K. (1983). L'idiot chinois: Initiation élémentaire à la lecture intelligible des caractères chinois. Paris : Payot.

Sartre J.-P. (1948). Qu'est-ce que la littérature ?, Paris : Gallimard.

Schmitt A. (2012). De la poétique cognitive et de ses (possibles) usages. Poétique, $n^{\circ} 170,143-162$. URL : https://www.cairn.info/revue-poetique-2012-2-page-143.htm

Sun H. (1990-1991). Cours de Français Accéléré I et II. Pékin : The Commercial Press.

Tompkins J. P. (1980). Reader-Response Criticism: From Formalism to Post-Structuralism. Baltimore : The Johns Hopkins University Press.

Tsur R. (2012). De la poétique cognitive et de ses (possibles) usages. Poétique, $n^{\circ} 170$.

Vallat, C. (2016). Impact du contexte d'enseignement/apprentissage sur la formation et les stratégies enseignantes en classe de Français Langue Etrangère (FLE), en milieu universitaire chinois. Recherches en didactique des langues et des cultures, 13-1, 1-18, URL : http://journals.openedition.org/rdlc/569 ; DOI : 10.4000/rdlc.569. 